\section{Wearable Textile Antenna in Substrate Integrated Waveguide Technology}

\author{
R. Moro, S. Agneessens, H. Rogier, and M. Bozzi
}

A novel wearable substrate integrated waveguide antenna fabricated entirely from textile materials is presented. The cavity-backed slot antenna operates in the $2.45 \mathrm{GHz}$ industrial, scientific and medical band, for short range communication between rescue workers. A prototype of the antenna was fabricated and tested: good performance was obtained in terms of input matching and radiation pattern. Moreover, measurements performed on the antenna after bending and integration into clothing indicate high robustness against deformation and low influence of the human body on antenna performance, making the design well-suited for on-body use.

Introduction: The design of wearable components and antennas for wireless applications received significant attention [1] in the past decade. In particular, localization and tracking of fire-fighters in rescue operations, as well as communication in critical conditions, require lowcost and efficient systems that are comfortable to wear. Therefore, components and systems should be low-profile, light-weight and mechanically flexible, and the adopted substrates need to be flame resistant and water repellent. The textile fabrics typically applied in firefighter garments represent good candidates for implementing antennas that are suitable for integration into protective garments. A variety of patch antennas on textile, operating in the $2.4-2.4835 \mathrm{GHz}$ industrial, scientific and medical (ISM) band, were proposed [1]. These antennas require a sufficiently large ground plane to limit the influence of the human body on the antenna's radiation characteristics. In addition, antenna performance should remain stable when the textile patch is subjected to bending [2].

In this Letter, a textile cavity-backed slot antenna in substrate integrated waveguide (SIW) technology is presented. To the authors' best knowledge this is the first implementation of a microwave SIW structure with textile materials. Cavity-backed antennas offer several advantages, such as suppression of unwanted surface waves and lower sensitivity for on-body operation, as well as a high front-to-back ratio [3]. SIW technology was chosen as a simple cost-effective fabrication process, which is already well-developed for printed circuit boards [4]. Furthermore, it allows easy integration of passive and active components onto the antenna [5], thus permitting the realization of complete systems on a textile carrier.

An SIW cavity-backed slot antenna in textile materials was designed, fabricated and experimentally verified. The structure exhibits compact size and good flexibility, thanks to the use of eyelets as metalized holes implementing the SIW cavity. On-body measurements were performed, and the effect of bending was investigated to evaluate the antenna performance under realistic operating conditions.

Antenna Design: The substrate adopted for the realization of the SIW cavity-backed antenna is a $3.94 \mathrm{~mm}$ thick closed cell expanded rubber protective foam typically used in fire-fighter suits, with dielectric permittivity $\varepsilon_{r}=1.575$ and loss tangent $\tan \delta=0.0238$, characterized at $2.45 \mathrm{GHz}$ [6]. Moreover, the top and bottom metal layers of the SIW structure are implemented by using Flectron ${ }^{\circledR}$, a conductive fabric with surface resistivity $R_{\mathrm{s}}=0.18 \Omega / \mathrm{sq}$ at $2.45 \mathrm{GHz}$.

The antenna consists of a square SIW cavity with a slot cut out in the top metal layer (Fig. 1a). A $50-\Omega$ grounded coplanar waveguide (GCPW) in the bottom metal layer was adopted as a feed line (Fig. 1b). The dimensions of the cavity were selected in order to excite the $\mathrm{TE}_{120}$ cavity mode at $2.45 \mathrm{GHz}$. The dog-bone shape of the slot allows to fit the slot in the top wall of the SIW cavity. Slot length and position were chosen for optimal coupling with the cavity mode: the best antenna performance was achieved with the slot slightly offset $(0.6 \mathrm{~mm})$ with respect to the centre of the cavity. Moreover, the length of the GCPW was optimized to improve the input matching.

The computer-aided design of the antenna was performed by using the commercial full-wave simulator Ansys HFSS.
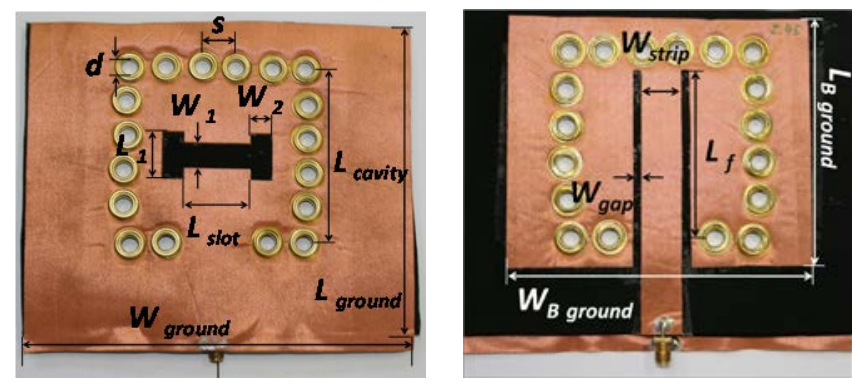

$a$

$b$

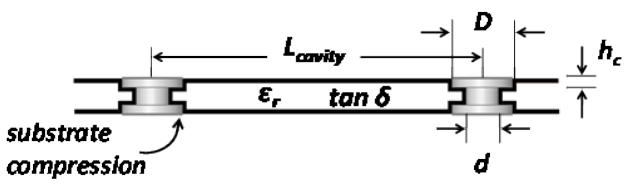

Fig. 1 SIW cavity-backed slot antenna.

a Top view

$b$ Bottom view

c Side view

Fabrication Process: First, the SIW textile antenna is assembled by gluing the two conductive fabrics onto the textile substrate by means of adhesive sheets, paying particular attention to the alignment of the different layers. Second, the structure is perforated to implement the via holes with diameter $d=7 \mathrm{~mm}$ and longitudinal spacing $s=14 \mathrm{~mm}$. Finally, eyelets are fixed in the holes. This solution permits to preserve the flexibility of the substrate.

A first prototype of the antenna was fabricated and tested. Compared to the simulation, the measured return loss exhibited an upward frequency shift. This discrepancy was attributed to the compression of the textile substrate and the conductive fabrics, due to the pressure of the heads of the eyelets (Fig. 1c). Consequently, the dimension of the cavity was reoptimized (Table I), taking into account this compression effect, and a new prototype of the antenna was fabricated.

Table 1 Dimensions of the re-optimized SIW cavity-backed antenna.

\begin{tabular}{|c|c|c|c|c|c|}
\hline parameter & $\begin{array}{c}\text { dimension } \\
{[\mathrm{mm}]}\end{array}$ & parameter & $\begin{array}{c}\text { dimension } \\
{[\mathrm{mm}]}\end{array}$ & parameter & $\begin{array}{c}\text { dimension } \\
{[\mathrm{mm}]}\end{array}$ \\
\hline$L_{\text {cavity }}$ & 71.5 & $W_{\text {strip }}$ & 15 & $L_{\text {ground }}$ & 126.5 \\
\hline$L_{\text {slot }}$ & 26.2 & $W_{\text {gap }}$ & 3 & $W_{\text {ground }}$ & 131.5 \\
\hline$L_{1}$ & 19.1 & $d$ & 7 & $L_{B \text { ground }}$ & 91.5 \\
\hline$W_{1}$ & 10.5 & $D$ & 13 & $W_{B \text { ground }}$ & 111.5 \\
\hline$W_{2}$ & 8.7 & $s$ & 14 & & \\
\hline$L_{f}$ & 64.6 & $h_{c}$ & 1 & & \\
\hline
\end{tabular}

Experimental Verification of the Antenna Performance: The input matching with respect to $50 \Omega$ was validated under different operating conditions of the antenna.

First, the antenna was measured standalone in an anechoic chamber. The simulated and measured reflection coefficients are shown in Fig. 2. The return loss is larger than $10 \mathrm{~dB}$ in the complete ISM band thanks to a resonance at $2.45 \mathrm{GHz}$ and a bandwidth of $165 \mathrm{MHz}$. The measured radiation patterns of the antenna in E-plane and H-plane are shown in Fig. 3. The measured co-polarized radiation patterns in both planes and the cross-polarization component in the H-plane agree very well with the simulations. Conversely, there is a small discrepancy for the crosspolarization component in the E-plane, which is extremely low in the simulation. The antenna gain at broadside was found to be $3.21 \mathrm{dBi}$ in the simulation and $3.9 \mathrm{dBi}$ in the measurement, with a measured frontto-back ratio of $19.7 \mathrm{~dB}$. Simulations yield an antenna efficiency of $52 \%$, compared to a measured efficiency of $68 \%$.

Second, the antenna is deployed on the human body, integrated into the backside of a fire-fighter jacket worn by a person. In order to avoid direct contact with the skin, the antenna was integrated between the waterproof aramid inner liner of the jacket and the moisture/thermal barrier. As shown in Fig. 2, the presence of human body does not affect the input matching significantly. Moreover, a similar radiation pattern is 
measured as in stand-alone conditions (Fig. $3 b$ ), with an increase of the measured gain at broadside $(4.9 \mathrm{dBi})$, attributed to the wider ground plane caused by the presence of the body. The on-body antenna efficiency was measured to be $60 \%$.

Finally, the influence of bending the antenna along the H-plane was investigated, to simulate the integration of the antenna on the arm of a fire-fighter jacket. This effect of bending results in a small upwards shift of the resonance, as shown in Fig. 2, for a bending diameter of 10 $\mathrm{cm}$. Even under this operation condition, the antenna meets the specifications of the ISM band.

Conclusion: A cavity-backed antenna in substrate integrated waveguide technology, realized completely in textile materials was designed, fabricated and validated. The SIW-on-textile integration results in an antenna exhibiting high robustness against bending, low influence of human body and high front-to-back ratio. In addition, this structure is light-weight, flexible and low-cost, thus making this antenna well-suited for on-body use.

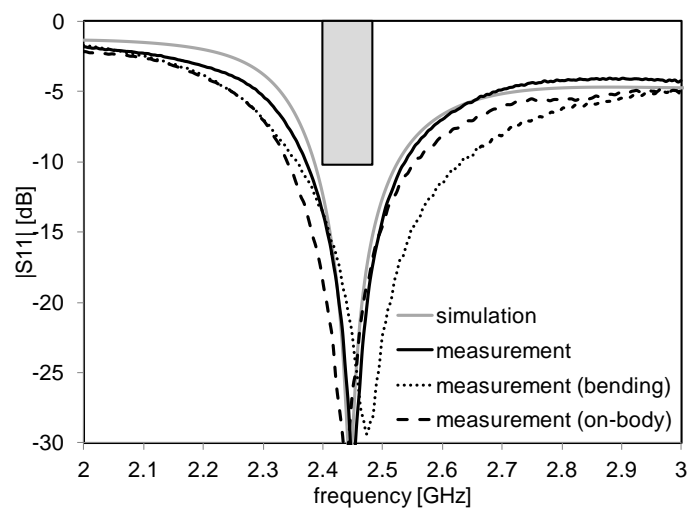

Fig. 2 Matching characteristics of the SIW cavity-backed slot antenna.
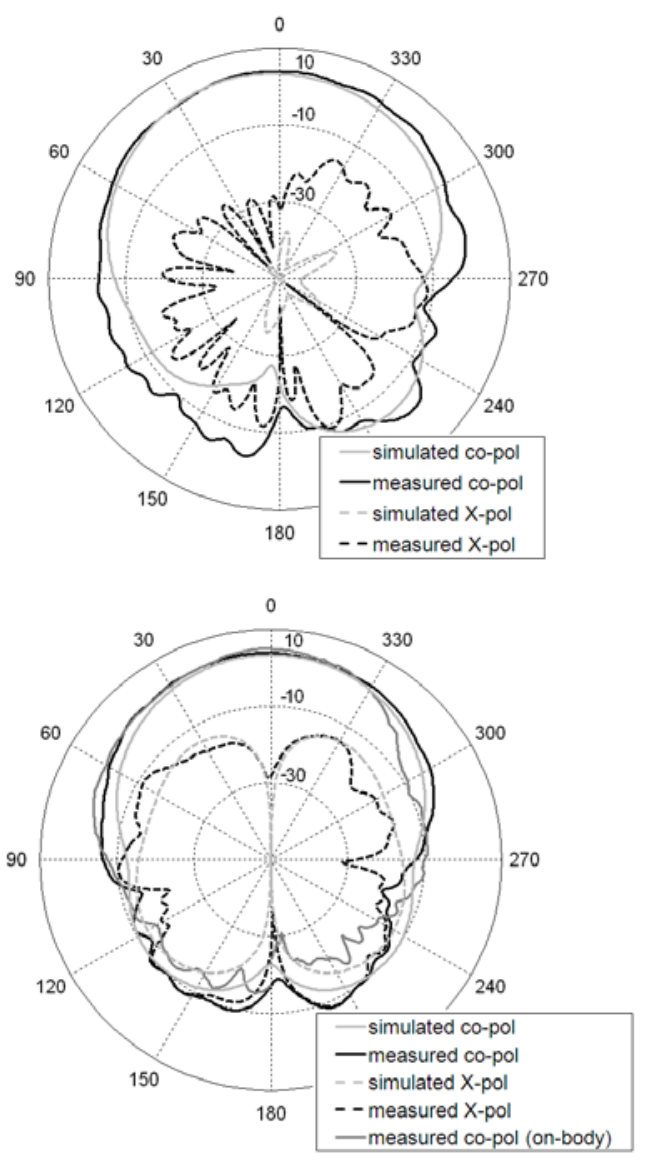

Fig. 3 Radiation pattern [dBi] of the SIW cavity-backed slot antenna. a E-plane $b$ H-plane
Acknowledgments: This work was performed in the framework of COST Action IC0803 (RFCSET).

R. Moro, M. Bozzi (Department of Electronics, University of Pavia, Pavia, Italy).

E-mail: riccardo.moro@unipv.it, maurizio.bozzi@unipv.it

S. Agneessens, H. Rogier (Department of Information TechnologyIMEC, Ghent University, Ghent, Belgium)

E-mail: sam.agneessens@intec.ugent.be, hendrik.rogier@ugent.be

\section{References}

1 Hertleer, C., Rogier, H., Vallozzi, L., and Van Langenhove, L.: ‘A Textile Antenna for Off-Body Communication Integrated Into Protective Clothing for Firefighters', IEEE Trans. Antennas Propag., 2009, 57, (4), pp. 919-925.

2 Boeykens F., Vallozzi L., and Rogier, H.: 'Cylindrical Bending of Deformable Textile Rectangular Patch Antennas', Inter. Journal Antennas Propag., 2012, Article ID 170420, pp. 1-11.

3 Guo, Q.L., Zhi, F.H., Lin, X.D., Ling, L.S.: 'Planar Slot Antenna Backed by Substrate Integrated Waveguide Cavity’, IEEE Antennas Wireless Propag. Lett., 2008, 7, (1), pp. 236-239.

4 Bozzi, M., Georgiadis, A., and Wu, K.: 'Review of Substrate Integrated Waveguide (SIW) Circuits and Antennas', IET Microwave, Antennas Propag., 2011, 5, (8), pp. 909-920.

5 Giuppi, F., Georgiadis, A. , Collado, A., Bozzi, M., and Perregrini L.: 'Tunable SIW Cavity Backed Active Antenna Oscillator', IET Electronics Letters, 2010, 46, (15), pp. 1053-1055.

6 Declercq, F., Rogier, H., and Hertleer, C.: 'Permittivity and loss tangent characterization for garment antennas based on a new matrixpencil two-line method', IEEE Trans. Antennas Propag., 2008, 56, (8), pp. 2548-2554. 